\title{
Analysis of networks of host proteins in the early time points following HIV transduction
}

\author{
Éva Csősz ${ }^{1 *} \mathbb{D}$, Ferenc Tóth ${ }^{2}$, Mohamed Mahdi ${ }^{2}$, George Tsaprailis ${ }^{3,4}$, Miklós Emri5 ${ }^{5+}$ and József Tőzsér ${ }^{1,2^{*+}}$
}

\begin{abstract}
Background: Utilization of quantitative proteomics data on the network level is still a challenge in proteomics data analysis. Currently existing models use sophisticated, sometimes hard to implement analysis techniques. Our aim was to generate a relatively simple strategy for quantitative proteomics data analysis in order to utilize as much of the data generated in a proteomics experiment as possible.

Results: In this study, we applied label-free proteomics, and generated a network model utilizing both qualitative, and quantitative data, in order to examine the early host response to Human Immunodeficiency Virus type 1 (HIV1). A weighted network model was generated based on the amount of proteins measured by mass spectrometry, and analysis of weighted networks and functional sub-networks revealed upregulation of proteins involved in translation, transcription, and DNA condensation in the early phase of the viral life-cycle.

Conclusion: A relatively simple strategy for network analysis was created and applied to examine the effect of HIV1 on host cellular proteome. We believe that our model may prove beneficial in creating algorithms, allowing for both quantitative and qualitative studies of proteome change in various biological and pathological processes by quantitative mass spectrometry.
\end{abstract}

Keywords: Weighted network, Quantitative proteomics, Host response, HIV-1

\section{Background}

Utilization of state-of the art proteomics methods can generate thousands of data points, and extensive information on proteins present in the sample can be obtained. High-resolution shotgun proteomics can provide both qualitative and quantitative information about proteins, and can be applied in an unbiased way to study the complete proteome $[1,2]$. Despite the high amount of data available, it is sometimes difficult to acquire relevant biological information, in which case sophisticated analytical methods and capable software are needed [3].

Network analysis is widely used in biological data analysis for examination of transcriptomic, proteomic or metabolomic datasets [4-6], and for analyzing interactions

\footnotetext{
*Correspondence: cseva@med.unideb.hu; tozser@med.unideb.hu ${ }^{+}$Miklós Emri and József Tőzsér contributed equally to this work.

${ }^{1}$ Proteomics Core Facility, Department of Biochemistry and Molecular Biology, Faculty of Medicine, University of Debrecen, Egyetem ter 1., Debrecen 4032, Hungary

Full list of author information is available at the end of the article
}

between various molecules $[7,8]$. In the cellular environment, most of the proteins exert their biological function as part of a complex, or in the form of interactions with other proteins, therefore, application of protein-protein interaction (PPI) analysis methods is advantageous [9]. PPI networks can provide a new layer of information, allowing for the utilization of currently available data, in addition to possibly unravelling hidden biological phenomena $[10,11]$.

New concepts on network analysis are emerging helping the understanding of biological complexity [12], however, in most cases, only the presence or absence of the protein is considered, the available quantitative data can hardly be incorporated into the network analyses.

The replication cycle of human immunodeficiency virus-1 (HIV-1) is a complex, multi-step, and highly regulated process. The cycle typically begins with viral attachment to cell surface receptors, and ending with the production of infectious virions. Due to the multiple processes involved, the replication cycle has been 
classically divided into two distinct phases; the early and late phase. The early phase encompasses cell binding, fusion, internalization, uncoating, reverse transcription, as well as integration of the viral cDNA into the host genome. On the other hand, transcription of viral genome, export of viral RNA, assembly of virions at the plasma membrane, as well as budding and maturation of the released virions are parts of the late phase of the replication cycle $[13,14]$. While late phase events are relatively well characterized, the precise mechanism and regulation of early phase steps remain poorly understood.

Genomics and proteomics studies were carried out to investigate how HIV-1 hijacks the host cellular machinery, avoiding being sensed by host immune responses. siRNA screens were implemented to study the cellular genes and proteins required for HIV-1 infection [9, 15, 16], HIV-1 protein - host protein protein-protein interaction networks were generated, and the data were deposited in HIV-1 Human Interaction Database [17].

In case of HIV infection, the network-based examinations have identified perturbed host cellular systems; such as the proteasome and transcriptional regulation, and have revealed that HIV-1 preferably interacts with highly connected and central cellular proteins [18-20].

In this study, we have generated the protein expression profiles of cells during early HIV-1 infection using protein mass spectrometry, and integrated the acquired data with knowledge-based protein-protein interaction network to understand how cellular network is perturbed by HIV.

\section{Results}

Our aim was to analyze the proteomic landscape of the early stage of HIV-1 based lentiviral vector transduction. $293 \mathrm{~T}$ cells were infected with VSV-G pseudotyped HIV1 vector, and 0,4 and $12 \mathrm{~h}$ post-infection, cell lysates were harvested. Label-free proteomics was applied to examine protein-level changes. Duplicate samples for three time points were collected $(0,4$, and $12 \mathrm{~h}$ posttransduction) in case of virus transduced samples and in case of control, mock transduced samples. The collected 6 virus treated and 6 control samples were analyzed in duplicates, allowing for the measurement of two technical and two biological replicates for each time point.

The mass spectrometry proteomics data have been deposited into the ProteomeXchange Consortium [21] via the PRIDE partner repository with the dataset identifier PXD010436 and https://doi.org/10.6019/PXD010436.

Identified proteins (Additional file 1) were manually curated, and in the case of non-human or non-viral identifications, the sequences were verified. In many instances, they were mistakenly designated as non-human proteins, in which case it was corrected. In few instances, the non-human proteins could not be matched to any of the human or viral proteins, and consequently, these sequences were omitted from further analyses. The data for Rhodobacter capsulatus cytochrome c, bovine pancreatic trypsin inhibitor, bovine serum albumin and pig trypsin were kept to serve as reference for quantitative analyses, but were not used for further computations. The relative amount of proteins was computed based on spectral counting and in case of each protein the mean of the results of the four analyses corresponding to each condition was calculated (Additional file 2).

\section{Statistical analysis}

Firstly, a qualitative analysis was carried out to detect newly expressed or down-regulated proteins in the first 4 or $12 \mathrm{~h}$ after HIV-1 pseudovirion transduction. Only those proteins were considered for statistical analysis which could be quantified in at least 2 out of 4 replicates, and were not quantified in other conditions. HIST1H1E, HNRNPL, PRRC2A and TRIM28 were quantified only at $\mathrm{H} 04$, and there were no proteins quantified solely in H12 time point (Additional files 1, 2). HIST1H1E interacts with linker DNA between nucleosomes, and functions in DNA condensation, HNRNPL and TRIM28 play a role in translation and transcription, while PRRC2A plays a role in inflammatory processes.

Some of the proteins were quantified in all time points except H12. These include ALYREF, CCDC86, CSDA, COX5A, HN1, MYL6, PPIF, SEPT2, SRSF6, TCOF1, and TPM3 (Additional file 1). These proteins participate in RNA binding (ALYREF, CCDC86, SRSF6, TCOF1), DNA binding (CSDA), protein folding (PPIF), energy generation (COX5A), signalization (HN1) and cytoskeleton assembly (MYL6, SEPT2, TPM3).

In order to examine changes in the amount of proteins, statistical analysis was carried out (Additional file 3). The amount of CSDA, EEF1A1, EEF1D, HN1, NPM1, PGAM1 and SRSF6 increased significantly, while that of HIST1H1D and HSPA5 significantly decreased in H04 (Fig. 1). It is interesting to note that after peaking in H04, CSDA, HN1 and SRSF6 were not quantified in H12. In $\mathrm{H} 12$, compared to $\mathrm{C} 12$, the amount of COX6B1 and PDIA3 increased, while that of EEF2 and GAPDH decreased significantly (Fig. 1). When the function of proteins showing statistically significant changes was examined, we observed an increase in the amount of proteins implicated in RNA binding in H04, and an overall decrease in their amount in H12.

\section{Network analysis}

To broaden our insight, and to better understand the possible functional associations of protein changes upon HIV-1 pseudovirion transduction, we have searched for the available protein-protein interactions of the quantified proteins in our datasets. For evaluation of the 


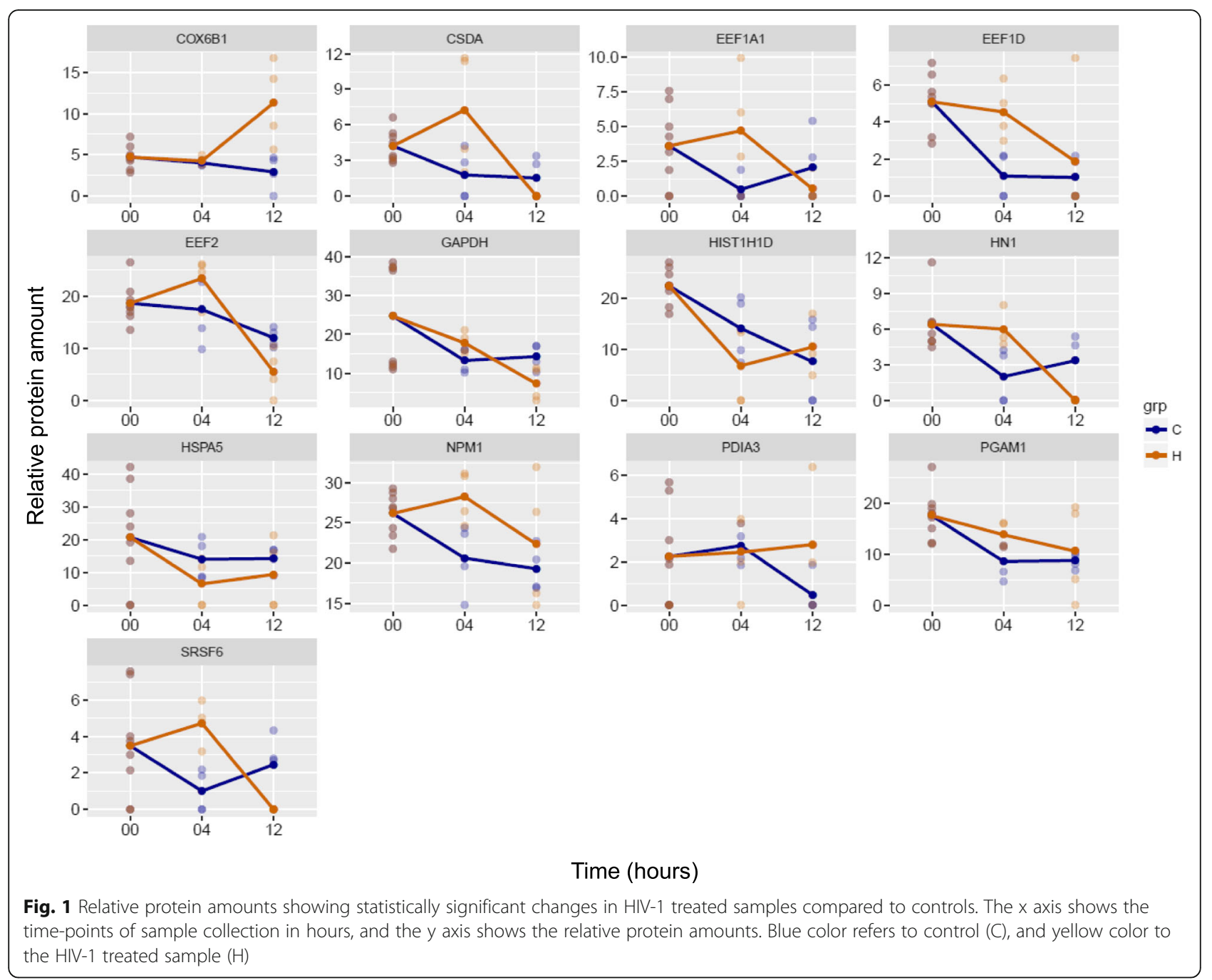

interactions, the STRING database was used, which contains information on known and predicted, direct physical, and indirect functional protein-protein interactions [22]. Only interactions which were of high confidence (interaction score in STRING database >0.95) were used. Initially, five binary interaction networks were generated: NW0 combined proteins from mock- and viriontreated cell lysates collected at 0 time-point, C04 and $\mathrm{C} 12$ networks contained proteins from the mock-treated cells collected $4 \mathrm{~h}$ and $12 \mathrm{~h}$ post-infection, respectively, and the $\mathrm{H} 04$ and $\mathrm{H} 12$ networks contained proteins from the HIV-1 transduced cells collected at 4 and $12 \mathrm{~h}$ timepoints, respectively (Fig. 2). The number of nodes and the number of edges of the networks show a decreasing trend over time, with a marked shrinkage in H12.

These binary networks provide information solely on the possibility of interaction between two proteins (Fig. 2, Fig. 3a, b), hence, in order to gain more realistic information, protein amounts measured by spectral counting were implemented into the network using a simple statistical model. In this way, binary edges were transformed into estimated protein pair's interaction intensities in the sample, which is proportional to the amounts of proteins participating in the interaction, and inversely proportional to the number of interactions (Fig. 3c). The weighted networks were examined, and the number of nodes $(\mathrm{N})$, edges $(\mathrm{E})$, network strength (S), edge density (D) and functional and non-functional edge ratio (R) were calculated (Fig. 4).

The number of nodes decreased significantly in H12, indicating network shrinkage in H12, observed in the binary network (Fig. 4a). The number of edges and network strength did not change in a statistically significant manner (Fig. 4b, c), however, edge density decreased significantly in H04 while increasing significantly in H12 (Fig. 4d). These changes indicate the presence of a less interactive network in H04, and a smaller; yet more active, PPI network in H12 (Fig. 4a, d).

Next, we were eager to analyze the functionality of the networks, and hence, we generated functional subnetworks of proteins belonging to GO terms. All the 


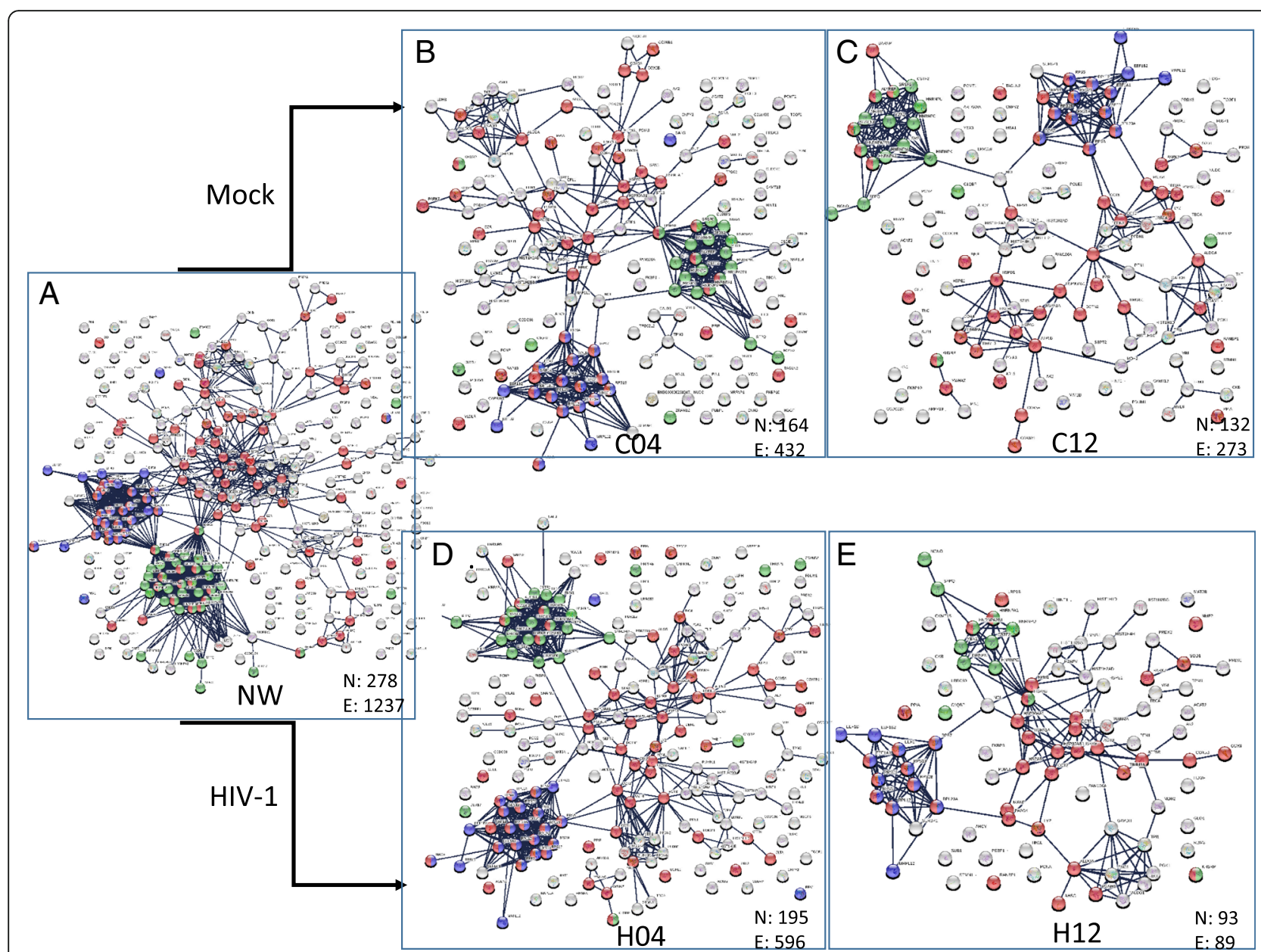

Fig. 2 Protein-protein interaction network of the proteins quantified in each condition. The PPI networks were generated by STRING (confidence 0.95) using the list of quantified proteins presented in Additional file 2 in case of each condition. The number of nodes $(\mathrm{N})$ and the number of edges (E) according to STRING in case of each network is indicated. a. PPI network of proteins in the $0 \mathrm{~h}$ time point (NW), b. PPI network of proteins in the $4 \mathrm{~h}$ time point in control, mock-transduced cells (C04). c PPI network of proteins in the $12 \mathrm{~h}$ time point in control, mocktransduced cells (C12). d. PPI network of proteins in the $4 \mathrm{~h}$ time point in HIV vector-transduced cells (H04). e. PPI network of proteins in the $12 \mathrm{~h}$ time point in HIV vector- transduced cells (H12). Red dots represent proteins belonging to transport GO term, blue dots indicate proteins having a role in translation, while green dots indicate proteins with a role in RNA splicing according to the functional enrichment analysis provided by STRING

Molecular Function, Biological Process and Cellular Component GO terms listed as enriched by STRING in C04, $\mathrm{H} 04, \mathrm{C} 12$ and $\mathrm{H} 12$; where at least 10 protein per GO function in any of the networks were present, were considered. To visualize network changes, the GO.0044765 term was chosen randomly (Fig. 3d), and the change of this sub-network was visualized in all time points (Fig. 5). Proteins present in a given GO term listed as enriched by STRING were considered as being part of the functional sub-network (f), whereas proteins not being part of the specific GO term, were considered as non-functional (n) proteins. Three types of interactions were analyzed: i) interactions between proteins belonging to functional subnetworks (f), ii) interactions between proteins not belonging to functional sub-network (n), and iii) interactions between functional and non-functional proteins (c - cross) (Fig. 3d). In order to better understand the changes, a statistical approach was applied, and the following network parameters were calculated: in case of each functional (f) network of proteins belonging to a specific GO term, the $\mathrm{Nf}$, Ef, Sf, Df, and Rf, while for non-functional (n) proteins the Nn, En, Sn, Dn, and Rn network parameters were calculated. In case of interactions between the functional and non-functional proteins (c) the Ec, Sc, Dc, and Rc network parameters were calculated (Additional file 4).

According to our hypothesis, those GO functions or functional sub-networks might be responsible for the changes induced by HIV-1, where the parameters in the functional network change significantly, whereas in the non-functional network, no statistically significant changes 

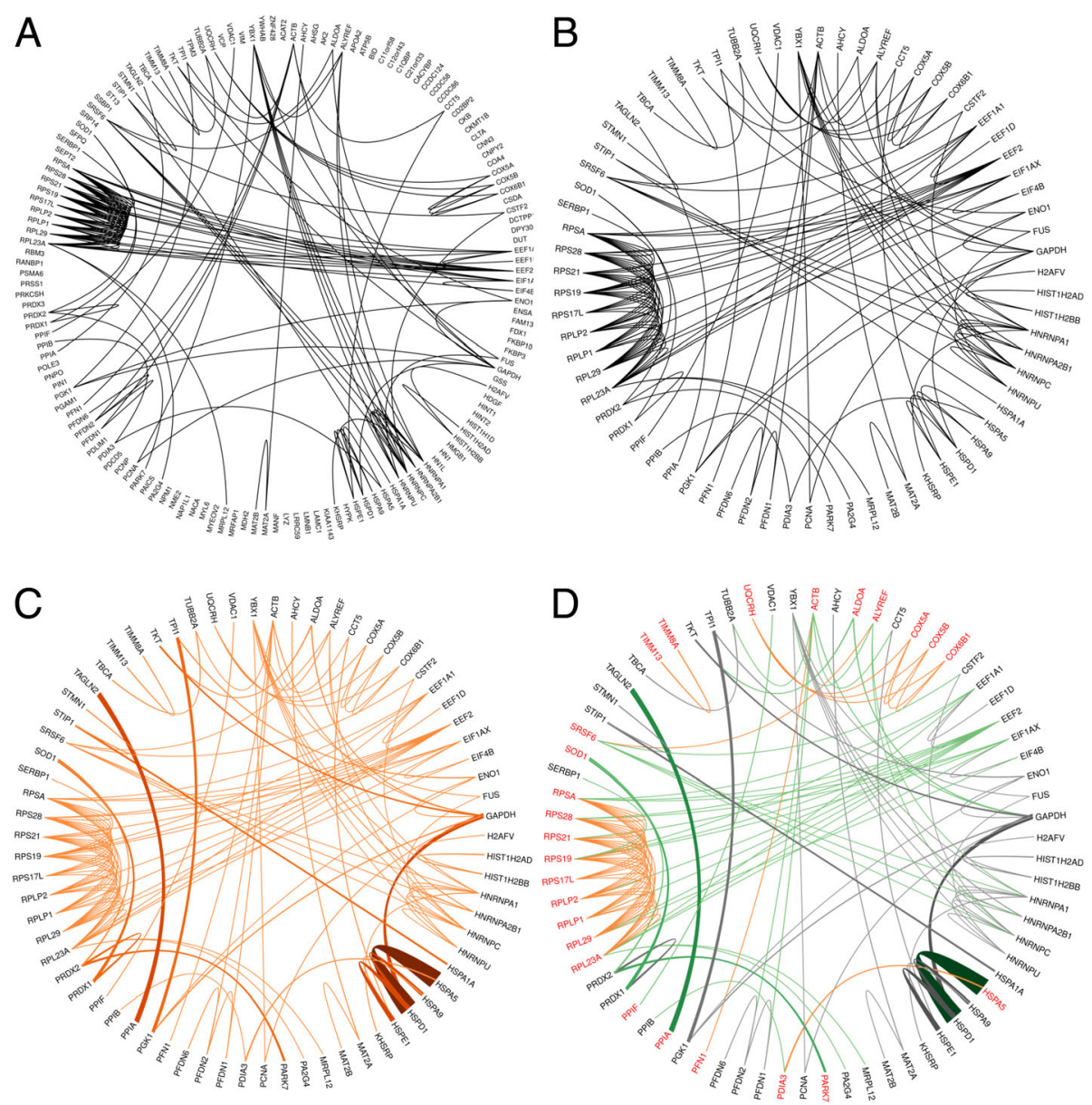

Fig. 3 Network generation pipeline. Representative network drawn by circlize, showing data for sample1 and interactions generated by STRING. a. Binary network containing all the identified proteins arranged in alphabetical order on the external ring of the circular plot. Thin black curves show the possible interactions generated by STRING. b. Binary network containing only the proteins with interactions. The isolated proteins (i.e. without any connection) were eliminated. Thin black curves show the possible interactions generated by STRING. c. Weighted network containing the interacting proteins. Orange lines represent interactions, the higher the intensity of the color and thickness of the line the higher the interaction strength. d. Weighted network with functional feature. A randomly selected GO function (GO:0044765) is used to illustrate the functional network. Red proteins are part of the functional sub-network, while black proteins are not, being considered as non-functional proteins. The weighted interactions are color-coded according to the protein-pair classification: functional - functional interactions are orange, non-functional - non-functional interactions are gray, and functional - non-functional interactions are green. The interaction strength is represented by the intensity of the color and thickness of the line: the higher the intensity of the color and the thickness of the line, the higher the interaction strength

are shown. At the same time, those GO functions where the parameters in the functional network do not change in a statistically significant manner, yet do so in the nonfunctional sub-networks, are thought to not explain the changes related to HIV-1 transduction.

After statistical analysis and FDR correction of the results (Additional file 5), in case of some GO terms, statistically significant differences were observed. No significant difference in edge and strength values were observed in any of the functional sub-networks (Ef and $\mathrm{Sf}$ ), and the number of nodes was significantly reduced in $\mathrm{H} 12$ only in the case of 5 functional sub-networks (Additional file 6). Considering edge density (D) and ratio (R), only those GO terms were further considered where (i) the significant difference was present only in the functional sub-network (Df and Rf, respectively) and (ii) where the significant difference was present both in the functional sub-network (Df and Rf) and in the cross network (Dc and Rc) (Additional file 6). According to our hypothesis, proteins belonging to the GO terms listed in Table 1 and Table 2, are responsible for the changes of cellular proteome observed in the $\mathrm{H} 04$ and H12 networks in response to HIV-1 transduction. In H04 sample, an increase in the node number (proteins present in the network) was observed, however, this increase was not significant. In the same time, a global 


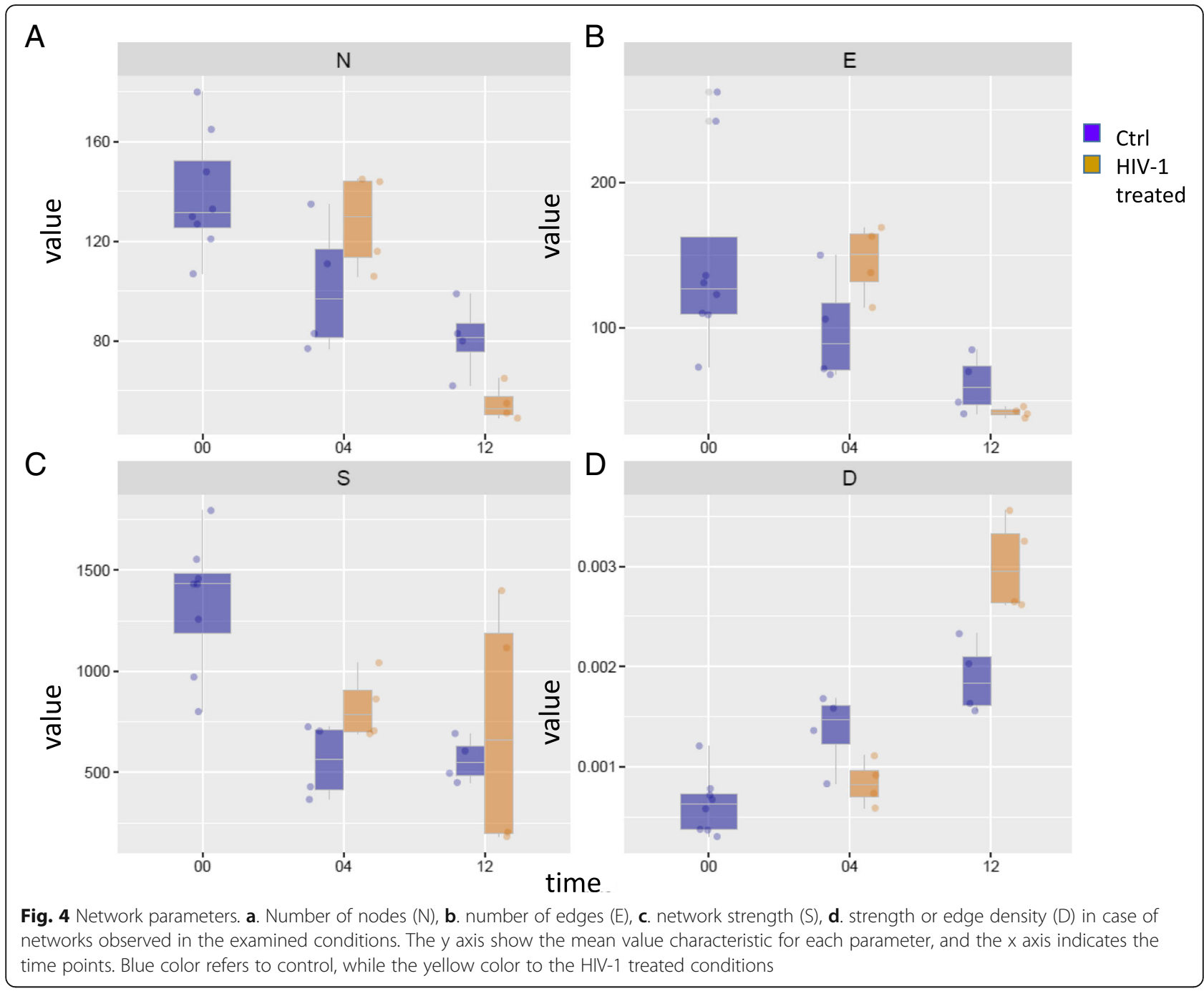

decrease in interactivity; represented by the number of edges, was noticed. Proteins which might be responsible for this reduced interactivity belong to the RNA processing-related functions (splicing, RNA synthesis, RNA catabolism, translation, transcription), regulation of cell death, regulation of cellular response to stress, viral life cycle (viral gene expression, viral transcription, viral life cycle) and protein localization, and some very general GO terms; such as protein binding, cellular macromolecular biosynthetic process, purine nucleotide binding, organic substance transport, etc. (Table 1). In spite of the reduced global interactivity, some functional sub-networks; such as viral process, protein kinase binding, multi-organism process, de novo protein folding and protein complex subunit organization, show significantly increased interactivity (Table 1 ).

In H12, a statistically significant reduction of the node numbers and shrinkage of the network; along with a significant increase in interactivity, was observed (Fig. 4). The proteins responsible for the increased interactivity (increased Df and Rf values) belong to RNA binding, RNA catabolic process, viral life cycle, viral process, negative regulation of cell death, de novo posttranslational protein folding, protein complex subunit organization, and cellular metabolic process, etc. (Table 2). The cell junction and the myelin sheet GO terms also appear in H12, however, when proteins belonging to these GO terms were examined, it was found that they are part of more general GO terms from the list; such as intracellular non-membranebounded organelle or nucleus, extracellular space, etc. In case of biosynthetic process functional sub-network (GO.0009058), a decrease in the Rf was observed.

\section{Discussion}

Genome-wide RNA interference-based screens were carried out to evaluate more than 20,000 human gene products to determine their alteration in HIV infection [23, 24]. A previous study showed an overall downregulation of cellular genes encoding for nuclear proteins, and genes involved in DNA replication and 

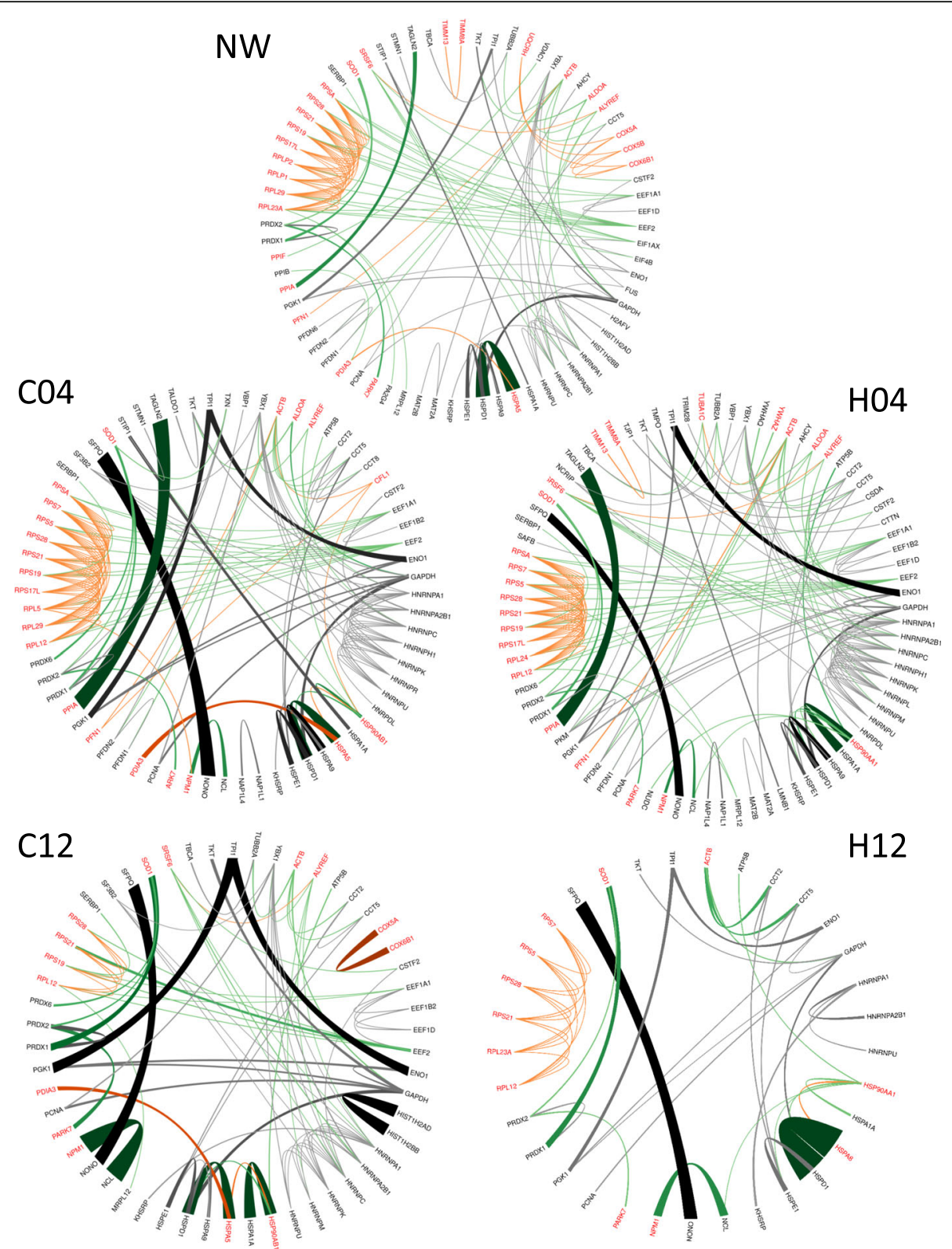

Fig. 5 Network changes visualized in case of a representative functional sub-network. The representative figure shows the changes in weighted networks in case of proteins belonging to randomly selected GO:0044765 GO term. NW represents the weighted PPI network of interacting proteins in the $0 \mathrm{~h}$ time point, C04 and C12 correspond to PPI networks of interacting proteins in the $4 \mathrm{~h}$ and $12 \mathrm{~h}$ time point, respectively, in control, mock-transduced cells. $\mathrm{H} 04$ and $\mathrm{H} 12$ represent the weighted PPI network of interacting proteins in the $4 \mathrm{~h}$ and $12 \mathrm{~h}$ time point, respectively, in HIV vector-transduced cells. Red proteins are part of the functional sub-network, while black proteins are non-functional proteins. The weighted interactions are color-coded according to the protein-pair classification: functional - functional interactions are orange, nonfunctional - non-functional interactions are gray, and functional - non-functional interactions are green. The interaction strength is represented by the intensity of the color and thickness of the line: the higher the intensity of the color and the thickness of the line, the higher the interaction strength

protein synthesis in the early stages of the early phase of viral infection [25], in a pattern that was confirmed by our analysis (Table 1). Upregulation of cellular genes was only found to occur at a later time point, peaking at $22 \mathrm{~h}$ post-infection, additionally, analysis on $\mathrm{T}$ cells showed that the most profound changes in cellular proteome appear $24 \mathrm{~h}$ after infection, at time points related to the late phase of infection [26].

It was found that up to 300 host cellular genes were involved in the life cycle of HIV-1, and while the identity of the genes was divergent among different studies, they were found more or less to belong to similar pathways 
Table 1 List of GO terms with significantly different changes in the functional sub-network in H04

\begin{tabular}{|c|c|c|c|}
\hline GO code & GO term & Significantly changed parameter & Direction of change in $\mathrm{H} 04$ \\
\hline GO.0000184 & nuclear-transcribed mRNA catabolic process, nonsense-mediated decay & Df & decrease \\
\hline G0.0000228 & nuclear chromosome & Df & decrease \\
\hline G0.0000375 & RNA splicing, via transesterification reactions & Df & decrease \\
\hline GO.0000398 & mRNA splicing, via spliceosome & Df & decrease \\
\hline GO.0000956 & nuclear-transcribed mRNA catabolic process & Df & decrease \\
\hline GO.0003735 & structural constituent of ribosome & Df & decrease \\
\hline GO.0005198 & structural molecule activity & Df & decrease \\
\hline GO.0005515 & protein binding & Df & decrease \\
\hline GO.0005524 & ATP binding & Df, Dc & decrease \\
\hline GO.0005622 & intracellular & Df & decrease \\
\hline GO.0005737 & cytoplasm & Df & decrease \\
\hline GO.0005739 & mitochondrion & Df & decrease \\
\hline GO.0005840 & ribosome & Df & decrease \\
\hline GO.0006351 & transcription, DNA-templated & Df, Dc & decrease \\
\hline G0.0006366 & transcription from RNA polymerase II promoter & Df, Dc & decrease \\
\hline G0.0006367 & transcription initiation from RNA polymerase II promoter & Df & decrease \\
\hline GO.0006396 & termination of RNA polymerase II transcription & Df & decrease \\
\hline GO.0006397 & mRNA processing & Df & decrease \\
\hline G0.0006401 & RNA catabolic process & Df & decrease \\
\hline GO.0006402 & mRNA catabolic process & Df & decrease \\
\hline GO.0006412 & translation & Df & decrease \\
\hline G0.0006413 & translational initiation & Df, Dc & decrease \\
\hline GO.0006414 & translational elongation & Df, Dc & decrease \\
\hline G0.0006415 & translational termination & Df & decrease \\
\hline G0.0006417 & regulation of translation & Df, Dc & decrease \\
\hline G0.0006518 & peptide metabolic process & Df & decrease \\
\hline G0.0006605 & protein targeting & Df & decrease \\
\hline G0.0006614 & SRP-dependent cotranslational protein targeting to membrane & Df & decrease \\
\hline G0.0006732 & coenzyme metabolic process & Df, Dc & decrease \\
\hline G0.0006886 & intracellular protein transport & Df & decrease \\
\hline GO.0008104 & protein localization & Rf & decrease \\
\hline GO.0008152 & metabolic process & Df & decrease \\
\hline GO.0008380 & RNA splicing & Df & decrease \\
\hline GO.0009056 & catabolic process & Df & decrease \\
\hline GO.0009892 & negative regulation of metabolic process & Df & decrease \\
\hline G0.0009987 & cellular process & Df & decrease \\
\hline G0.0010468 & regulation of gene expression & Df & decrease \\
\hline G0.0010556 & regulation of macromolecule biosynthetic process & Df & decrease \\
\hline G0.0010608 & posttranscriptional regulation of gene expression & Df, Dc & decrease \\
\hline G0.0010941 & regulation of cell death & Df & decrease \\
\hline G0.0015031 & protein transport & Df & decrease \\
\hline G0.0016032 & viral process & Df & increase \\
\hline G0.0016043 & cellular component organization & Df & decrease \\
\hline GO.0016070 & RNA metabolic process & Df, DC & decrease \\
\hline
\end{tabular}


Table 1 List of GO terms with significantly different changes in the functional sub-network in H04 (Continued)

\begin{tabular}{|c|c|c|c|}
\hline GO code & GO term & Significantly changed parameter & Direction of change in $\mathrm{H} 04$ \\
\hline GO.0016071 & mRNA metabolic process & Df & decrease \\
\hline GO.0016482 & cytoplasmic transport & Df & decrease \\
\hline GO.0016604 & nuclear body & Df, Dc & decrease \\
\hline GO.0017076 & purine nucleotide binding & Df, Dc & decrease \\
\hline GO.0018130 & heterocycle biosynthetic process & Df, Dc & decrease \\
\hline GO.0019058 & viral life cycle & Df & decrease \\
\hline GO.0019080 & viral gene expression & Df & decrease \\
\hline GO.0019083 & viral transcription & Df & decrease \\
\hline GO.0019222 & regulation of metabolic process & Df & decrease \\
\hline GO.0019438 & aromatic compound biosynthetic process & Df, Dc & decrease \\
\hline GO.0019538 & aromatic compound biosynthetic process & Df & decrease \\
\hline GO.0019899 & enzyme binding & Df & decrease \\
\hline GO.0019900 & kinase binding & Df, Dc & increase \\
\hline GO.0019901 & protein kinase binding & Df & increase \\
\hline GO.0022607 & cellular component assembly & Df & decrease \\
\hline G0.0022626 & cytosolic ribosome & Df & decrease \\
\hline GO.0030554 & adenyl nucleotide binding & Df, Dc & decrease \\
\hline GO.0031625 & ubiquitin protein ligase binding & Df, Dc & decrease \\
\hline GO.0031974 & membrane-enclosed lumen & Df & decrease \\
\hline GO.0032550 & purine ribonucleoside binding & Df, Dc & decrease \\
\hline GO.0032553 & ribonucleotide binding & Df, Dc & decrease \\
\hline GO.0032555 & purine ribonucleotide binding & Df, Dc & decrease \\
\hline GO.0032774 & RNA biosynthetic process & Df, Dc & decrease \\
\hline GO.0034248 & regulation of cellular amide metabolic process & Df & decrease \\
\hline GO.0034613 & cellular protein localization & Rf & decrease \\
\hline G0.0034622 & cellular macromolecular complex assembly & Df & decrease \\
\hline GO.0034645 & cellular macromolecule biosynthetic process & Df & decrease \\
\hline GO.0034654 & nucleobase-containing compound biosynthetic process & Df, Dc & decrease \\
\hline GO.0034655 & nucleobase-containing compound catabolic process & Df & decrease \\
\hline GO.0035639 & purine ribonucleoside triphosphate binding & Df, Dc & decrease \\
\hline GO.0036094 & small molecule binding & Df, Dc & decrease \\
\hline GO.0042981 & regulation of apoptotic process & Df & decrease \\
\hline GO.0043065 & positive regulation of apoptotic process & Df & decrease \\
\hline G0.0043066 & negative regulation of apoptotic process & Df, Dc & decrease \\
\hline GO.0043226 & organelle & Df & decrease \\
\hline G0.0043227 & membrane-bounded organelle & Df, Dc & decrease \\
\hline GO.0043229 & intracellular organelle & Df & decrease \\
\hline GO.0043231 & intracellular membrane-bounded organelle & Df & decrease \\
\hline G0.0043233 & organelle lumen & Df & decrease \\
\hline GO.0044085 & cellular component biogenesis & Df & decrease \\
\hline G0.0044237 & cellular metabolic process & Df & decrease \\
\hline GO.0044238 & primary metabolic process & Df & decrease \\
\hline G0.0044248 & cellular catabolic process & Df & decrease \\
\hline GO.0044249 & cellular biosynthetic process & Df & decrease \\
\hline
\end{tabular}


Table 1 List of GO terms with significantly different changes in the functional sub-network in H04 (Continued)

\begin{tabular}{|c|c|c|c|}
\hline GO code & GO term & Significantly changed parameter & Direction of change in $\mathrm{H} 04$ \\
\hline GO.0044260 & cellular macromolecule metabolic process & Df & decrease \\
\hline G0.0044265 & cellular macromolecule catabolic process & Df & decrease \\
\hline GO.0044267 & cellular protein metabolic process & Df & decrease \\
\hline GO.0044271 & cellular nitrogen compound biosynthetic process & Df, Dc & decrease \\
\hline GO.0044391 & ribosomal subunit & Df & decrease \\
\hline GO.0044422 & organelle part & Df & decrease \\
\hline GO.0044424 & intracellular part & Df & decrease \\
\hline GO.0044444 & cytoplasmic part & Df & decrease \\
\hline GO.0044446 & intracellular organelle part & Df & decrease \\
\hline G0.0044451 & nucleoplasm part & Df, Dc & decrease \\
\hline GO.0044454 & nuclear chromosome part & Df & decrease \\
\hline GO.0044802 & single-organism membrane organization & Df, Dc & decrease \\
\hline GO.0045184 & establishment of protein localization & Df & decrease \\
\hline GO.0046907 & intracellular transport & Df, Dc & decrease \\
\hline GO.0051084 & de novo posttranslational protein folding & Df & increase \\
\hline GO.0051704 & multi-organism process & Df, Dc & increase \\
\hline GO.0060255 & regulation of macromolecule metabolic process & Df & decrease \\
\hline GO.0060548 & negative regulation of cell death & Df, Dc & decrease \\
\hline G0.0065003 & macromolecular complex assembly & Df & decrease \\
\hline GO.0070013 & intracellular organelle lumen & Df & decrease \\
\hline GO.0070972 & protein localization to endoplasmic reticulum & Rf & decrease \\
\hline GO.0071013 & catalytic step 2 spliceosome & Df & decrease \\
\hline GO.0071702 & organic substance transport & Df, Dc & decrease \\
\hline GO.0071704 & organic substance metabolic process & Df & decrease \\
\hline GO.0071705 & nitrogen compound transport & Df, Dc & decrease \\
\hline GO.0071822 & protein complex subunit organization & Df & increase \\
\hline GO.0071840 & cellular component organization or biogenesis & Df & decrease \\
\hline GO.0072594 & establishment of protein localization to organelle & Df & decrease \\
\hline GO.0080090 & regulation of primary metabolic process & Df & decrease \\
\hline GO.0080135 & regulation of cellular response to stress & Df, Dc & decrease \\
\hline GO.0090304 & nucleic acid metabolic process & Df, Dc & decrease \\
\hline GO.0097367 & carbohydrate derivative binding & Df, Dc & decrease \\
\hline GO.1901362 & organic cyclic compound biosynthetic process & Df, Dc & decrease \\
\hline GO.1901566 & organonitrogen compound biosynthetic process & Df & decrease \\
\hline GO.1901575 & organic substance catabolic process & Df & decrease \\
\hline GO.1901576 & organic substance biosynthetic process & Df & decrease \\
\hline GO.1902580 & single-organism cellular localization & Df & decrease \\
\hline
\end{tabular}

In case of each GO term where statistically significant changes were observed in network parameters in HIV-1 treated cells at $4 \mathrm{~h}$ time-point the GO identifier, the changed parameter and the direction of change is indicated

$[27,28]$. Network analysis is widely used in the examination of protein-protein interactions, providing information regarding protein changes on a different level, giving a more ample view of the alterations and perturbations of the biological systems as a result of a particular treatment. During analysis of PPIs, the presence or absence of a protein is evaluated, and the interactions, in light of existing evidence (ex. experimental data, literature search, computational methods), are displayed [29, 30]. STRING is a widely used, constantly updated, and expanding database of PPIs [22], used for the examination of verified, or potential interactions among 
Table 2 List of GO terms with significantly different changes in the functional sub-networks in $\mathrm{H} 12$

\begin{tabular}{|c|c|c|c|}
\hline GO code & GO term & Significantly changed parameter & Direction of change in $\mathrm{H} 12$ \\
\hline GO.0005759 & mitochondrial matrix & $\mathrm{Nf}$ & decrease \\
\hline GO.0003674 & molecular function & Df, Dc & increase \\
\hline GO.0003723 & RNA binding & $\mathrm{Rf}, \mathrm{Rc}$ & increase \\
\hline GO.0005488 & binding & Df, Dc & increase \\
\hline GO.0005615 & extracellular space & $\mathrm{Rf}, \mathrm{Rc}$ & increase \\
\hline GO.0005634 & nucleus & Df, Dc & increase \\
\hline GO.0005654 & nucleoplasm & Df, Dc & increase \\
\hline GO.0005739 & mitochondrion & Df & increase \\
\hline GO.0005743 & mitochondrial inner membrane & Df, Dc & increase \\
\hline GO.0005759 & mitochondrial matrix & Df & increase \\
\hline GO.0005856 & cytoskeleton & Df, Dc & increase \\
\hline G0.0006401 & RNA catabolic process & Df, Dc & increase \\
\hline G0.0006402 & mRNA catabolic process & Df, Dc & increase \\
\hline GO.0009058 & biosynthetic process & $\mathrm{Rf}, \mathrm{Rc}$ & decrease \\
\hline GO.0009987 & cellular process & Df, Dc & increase \\
\hline G0.0010467 & gene expression & Rf, Rc & increase \\
\hline G0.0016032 & viral process & Rf & increase \\
\hline G0.0016604 & nuclear body & $\mathrm{Nf}$ & decrease \\
\hline G0.0017076 & purine nucleotide binding & Df, Dc & increase \\
\hline GO.0019058 & viral life cycle & Df, Dc & increase \\
\hline G0.0022607 & cellular component assembly & Df & increase \\
\hline GO.0030054 & cell junction & Df & increase \\
\hline G0.0031966 & mitochondrial membrane & Df, Dc & increase \\
\hline GO.0031981 & nuclear lumen & Df, Dc & increase \\
\hline G0.0043066 & negative regulation of apoptotic process & Df & increase \\
\hline G0.0043209 & myelin sheath & Df & increase \\
\hline G0.0043232 & intracellular non-membrane-bounded organelle & Df & increase \\
\hline G0.0043933 & macromolecular complex subunit organization & Rf, Rc & increase \\
\hline G0.0044237 & cellular metabolic process & Df & increase \\
\hline G0.0044265 & mitochondrial nucleoid & Df, Dc & increase \\
\hline G0.0044428 & nuclear part & Df, Dc & increase \\
\hline G0.0044430 & cytoskeletal part & Df, Dc & increase \\
\hline G0.0044451 & nucleoplasm part & $\mathrm{Nf}$ & decrease \\
\hline G0.0044712 & single-organism catabolic process & $\mathrm{Nf}$ & decrease \\
\hline G0.0044765 & single-organism transport & Df, Dc & increase \\
\hline G0.0044822 & poly(A) RNA binding & $\mathrm{Rf}$ & increase \\
\hline G0.0051084 & de novo posttranslational protein folding & $\mathrm{Rf}, \mathrm{Rc}$ & increase \\
\hline G0.0051704 & multi-organism process & $\mathrm{Rf}, \mathrm{Rc}$ & increase \\
\hline G0.0060548 & negative regulation of cell death & Df & increase \\
\hline G0.0071013 & catalytic step 2 spliceosome & Df & increase \\
\hline G0.0071705 & nitrogen compound transport & $\mathrm{Nf}$ & decrease \\
\hline G0.0071822 & protein complex subunit organization & Rf & increase \\
\hline GO.1901575 & organic substance catabolic process & Df & increase \\
\hline
\end{tabular}


proteins of interests. These networks are rich in information on protein clusters and functions based on Gene Ontology (GO), however, enrichment of GO terms does not handle protein amounts, therefore, reflecting theoretical, rather than actual parameters. Meanwhile, the use of highly accurate mass spectrometry techniques provide analytical data that is wealthy in quantity as well as quality. There were few attempts made to introduce the quantitative data into the network analysis [31, 32]. In order to implement quantitative data into the PPI networks, instead of the widely used binary networks, a weighted network often utilized in information science [33] was used in this study. Taking into account the protein amount reflected by the normalized total spectra, instead of the probabilistic assumption [32], we choose a simple statistical model. In our model, the protein pair's interaction is proportional to their amount in the sample, and inversely proportional to the number of possible interactions listed in the PPI network generated by STRING for proteins present in the sample. After including the interaction density values as network edge weights; calculated by our method, we could determine a sort of weighed network parameters for the statistical investigation of network alterations.

In our study, we aimed at characterizing the cellular proteome changes in the early stage of HIV-1 infection, within the $0-12 \mathrm{~h}$ time interval. Generation of weighted networks, and analysis of functional sub-networks revealed that the dynamics of protein level changes in subnetworks is different in HIV-1 transduced samples $12 \mathrm{~h}$ post-infection. Expectedly, in the very early stages of infection, proteins involved in translation, transcription and DNA condensation were upregulated, notably HIST1H1E, HNRNPL, PRRC2A and TRIM28. Some other proteins; such as ALYREF, CCDC86, CSDA, COX5A, HN1, MYL6, PPIF, SEPT2, SRSF6, TCOF1, and TPM3, prominently associated with RNA binding, cytoskeleton assembly, and signaling were quantified in all time points except $\mathrm{H} 12$.

Examining the binary networks, two protein clusters could be observed. One comprising proteins having a role in translation and ribosome biogenesis, and the other containing proteins from the hnRNP family with a role in RNA splicing (Fig. 2). The functional subnetwork containing the ribosome component proteins did not show a statistically significant change, and with this, we can demonstrate on protein level the same findings observed by Kleinman et.al. at gene level, who could not observe statistically significant difference in case of genes having a role in ribosome biogenesis at $12 \mathrm{~h}$ time point [34]. Regarding the other cluster containing mainly hnRNP proteins, we could not observe a statistically significant change in network parameters among the different time points. However, literature data show that host
RNA splicing is altered upon HIV-1 infection, and the level of class $\mathrm{A} / \mathrm{B}$ and $\mathrm{H}$ of hnRNP proteins changes; initially decreased 6-12 days post infection, thereafter increased [35]. At the same time, it was shown that some proteins of this cluster; such as HNRNPH1, HNRNPU and SRSF6, are so called HIV-1 dependency factors [36] and are required by HIV-1. These data are derived from later time-points, as most of the experiments do not examine such early events at $4 \mathrm{~h}$ or $12 \mathrm{~h}$ post infection.

Considering the results of the analyses, based on the weighted networks, we could identify increased cellular metabolic processes comprising increased RNA binding and catabolism, cellular component assembly, along with increased viral process and inhibition of apoptosis (increased negative regulation of apoptotic process). RNA binding was shown to be increased upon RNA virus infection; Garcia-Moreno et al. observed an increased activity of RNA-binding proteins upon sindbis virus (SINV) infection at $18 \mathrm{~h}$ time point [37]. At the same time, they observed an increased binding of RNA binding proteins to viral RNAs. This implies a massive downregulation of the host mRNAs $18 \mathrm{~h}$ post infection, involving mainly the housekeeping genes [37]. In case of HIV-1 infection, global siRNA studies indicate that a statistically significant portion of the host factors participate in mRNA transport [18].

Cells infected with HIV-1 usually die by apoptosis, hence prevention of apoptosis might help maintain the viral reservoir in the host $[18,38]$. It was shown that a fraction of infected immune cells survive, highlighting the importance of escaping from apoptosis in the development of viral reservoirs [38]. A mixed pattern of upregulation and downregulation of genes involved in antiviral defense and cell death signaling were observed by Mohammadi et al. at early time points [24]. Inhibition of apoptosis increases the virus production in HIV-1 infected cells [39], and modulation of this system might be a good possibility for a therapeutic intervention [40].

Based on our data on the weighted networks, HSPA8 shows an increased interactivity in $\mathrm{H} 12$ datasets (Fig. 5a). HSPA8 and other members of the Hsp70 family play a key role during viral infection either as receptors for the virus, as chaperons aiding the protein folding, or as transporters between organelles [18, 41, 42].

Hijacking of the host system by HIV-1 is a complex phenomenon with early and late events. In the early phases of the viral infection, the virus utilizes cellular RNA and protein production machinery for its replication. It was observed that by $15 \mathrm{~h}$ post infection, all viral transcripts were produced by the cells, and $18 \mathrm{~h}$ after infection, the virus budding commences [24]. Chang et al.;. using next generation sequencing, observed a considerable viral mRNA level in infected cells $12 \mathrm{~h}$ post-infection [43]. In this sense, examining the host response $48 \mathrm{~h}[15,44]$ or 
6 days post-infection [45] cannot provide us with information on the very early events. Observations made by Kleinmann et al. analyzing the dataset generated by Chang et al., show that at $12 \mathrm{~h}$ post infection, the gene expression profiles are similar to the mock samples, and clear distinctions could only be made after $24 \mathrm{~h}$, highlighting the necessity of more sensitive methods for the examination of early events of HIV-1 infection.

It is challenging to properly compare our results to those presented in the scientific literature, since the commonly used starting time point examined is $48 \mathrm{~h}$ post infection, in case of HIV-1. However, considering the findings presented by different groups; either on HIV-1 or other RNA virus infections, our findings are in good agreement with previous studies analyzing transcriptomic and proteomic changes upon virus infection in these very early time points. The use of non-primary HIV-1 cell targets; such as HEK, and pseudotyped virions, and the application of datadependent sampling [46], may indeed limit interpretation of the results. The utilization of other cell types and data acquisition methods with higher reproducibility; such as parallel reaction monitoring [2] or data independent acquisition [47], might give more accurate input data. In spite of the above limitations, we believe that this model of proteomic data evaluation serves as a good starting point for further development of algorithms implementing not only qualitative, but also quantitative data generated in a given proteomic experiment, and that such a combination will undoubtedly aid in the understanding and deciphering of complex biological phenomena.

\section{Conclusion}

A weighted network model facilitating the use of both qualitative and quantitative data, acquired in a labelfree proteomics experiment was generated and applied to examine the early host response to HIV-1. Upregulation of proteins involved in translation, transcription and DNA condensation in the early phase of the viral life-cycle could be observed, highlighting the utility of our weighted PPI network data analysis approach. More studies are required to further demonstrate the utility of this new data-driven weighted network based analysis, and it should be noted that the current model has a serious limitation. The strength of different protein-protein interactions in the edge weight calculation; due to the lack of information, is not yet included. However, the applied weight-model can easily be extended to use this type of information as soon as any public database becomes available. We hope that this approach can open new ways for creating algorithms, allowing for both quantitative and qualitative studies of proteome change in various biological and pathological processes by quantitative mass spectrometry.

\section{Methods \\ Production of viral particles}

Viral particles were produced with some modifications of a previously utilized protocol [48]. Briefly, recombinant viruses were produced by transient transfection of 293 T cells (ATCC ${ }^{ø}$ CRL3216 $^{\text {max}}$ ) using pWOX-CMV-GFP (transfer vector plasmid), pMDLg/pRRE (packaging plasmid), pRSV.rev (Rev-coding plasmid), and pMD. G (VSV-G envelope protein-coding plasmid). Vectors were a kind gift from D. Trono (University of Geneva Medical School, Geneva, Switzerland) [49], and were subsequently modified by our research group [48]. Salmon sperm DNA (Sigma-Aldrich) was also added. Media containing virus particles was concentrated by Ultracel$100 \mathrm{~K}$ Amicon Ultra Centrifugal Filter (Millipore), and stored in $-70{ }^{\circ} \mathrm{C}$. Quantity of pseudovirions produced was assessed by measurement of reverse transcriptase (RT) activity using a colorimetric kit (Sigma-Aldrich, Roche).

\section{Transduction and sample collection}

$293 \mathrm{~T}$ cells in T-25 cell culture flasks were either mocktreated or transduced at 50\% confluency with $5 \mathrm{ng}$ RT equivalent of the HIV-based pseudovirions, in the presence of $4 \mu \mathrm{g} / \mathrm{ml}$ polybrene (Sigma-Aldrich), in $1 \mathrm{ml}$ total volume, and incubated at $37 \mathrm{C}^{\circ}$. After 0,4 , and $12 \mathrm{~h}$, cells were trypsinized for $10 \mathrm{~min}$, then washed tree times with ice-cold PBS to remove non-fused pseudovirion particles. The final pellet was suspended in $4 \mathrm{ml}$ lysis buffer (150 mM sodium chloride, 1.0\% Triton X-100, $0.5 \%$ sodium deoxycholate, $0.1 \%$ sodium dodecyl sulfate (SDS), and $50 \mathrm{mM}$ Tris) $\mathrm{pH} 8.0$, supplemented with cOmplete protease inhibitor cocktail (Sigma-Aldrich), incubated for $30 \mathrm{~min}$ at room temperature, centrifuged, and the supernatant was mixed with $24 \mathrm{ml}$ cold $(-20$ $\mathrm{C}^{\circ}$ ) acetone and stored at $-20 \mathrm{C}^{\circ}$ overnight.

\section{Mass spectrometry analysis}

The cleared cell lysates were acetone-precipitated with six volumes of cold acetone overnight. The precipitates were re-dissolved in $25 \mathrm{mM}$ ammonium bicarbonate (Sigma-Aldrich) and digested in-solution with trypsin [50]. The tryptic fragments were used for replicate LCMS/MS analyses at University of Arizona in Tucson, AZ, USA.

$500 \mathrm{ng}$ per $5 \mu \mathrm{L}$ injected protein lysate spiked with 300 fmol of Rhodobacter capsulatus cytochrome c T33 V mutant, was analyzed using a LTQ Orbitrap Velos mass spectrometer (Thermo Fisher Scientific) equipped with an Advion nanomate ESI source (Advion), after Omix (Agilent Technologies) C18 sample clean-up according 
to the manufacturer's instructions. Peptides were eluted from a C18 precolumn $(100-\mu \mathrm{m} \times 2 \mathrm{~cm}$, Thermo Fisher Scientific) onto an analytical column $(75-\mu \mathrm{m} \times 10 \mathrm{~cm}$, C18, Thermo Fisher Scientific) using a 165 min gradient of solvent A (water, $0.1 \%$ formic acid) and solvent B (acetonitrile, $0.1 \%$ formic acid). The flow rate was 500 $\mathrm{nl} /$ minute. Data-dependent analysis (DDA) was performed by the Xcalibur v 2.1.0 software [51] using a survey mass scan at 60,000 resolution in the Orbitrap analyzer scanning mass/charge 350-1600, followed by collision-induced dissociation tandem mass spectrometry (MS/MS) at 35 normalized collision energy of the 14 most intense ions in the linear ion trap analyzer. Precursor ions were selected by the monoisotopic precursor selection setting with selection or rejection of ions held to a $+/-10 \mathrm{ppm}$ window. Singly charged ions were excluded from MS/MS. Dynamic exclusion was set to place any selected $\mathrm{m} / \mathrm{z}$ on an exclusion list for $45 \mathrm{~s}$ after a single MS/ MS. Tandem mass spectra were searched against the UniprotKB/Swiss-Prot release available on December 12, 2014 without species restriction. At the time of the search, this database contained 459,734 entries. All MS/MS spectra were searched using Thermo Proteome Discoverer 1.3 (Thermo Fisher Scientific) considering fully tryptic peptides with up to 2 missed cleavage sites. Variable modifications considered during the search included methionine oxidation $(15.995 \mathrm{Da})$, and cysteine carbamidomethylation $(57.021 \mathrm{Da})$. The parent ion mass tolerance was $10 \mathrm{ppm}$, while the fragment tolerance was $0.8 \mathrm{Da}$. Proteins were identified at 99\% confidence with XCorr score cut-offs [52] as determined by a reversed database search. The protein and peptide identification results were validated with Scaffold v4.4.6. (Proteome Software Inc.) [1]. Peptide identifications were accepted if they had greater than $89 \%$ probability to achieve an FDR less than $0.1 \%$ by the Scaffold Local FDR algorithm. Protein identifications were accepted if they had greater than $99 \%$ probability and contained at least 2 identified peptides. Protein probabilities were assigned by the ProteinProphet algorithm [53]. Proteins that contained similar peptides and could not be differentiated based on MS/MS analysis alone were grouped to satisfy the principles of parsimony. Proteins sharing significant peptide evidence were grouped into clusters.

Protein quantification was done based on spectral counting; the quantitative values were generated by the Scaffold program based on the normalized total spectra. In case of protein clusters, each peptide was used only once for quantification for the first human protein in the cluster, as listed by Scaffold. All quantitative data were used for statistical analyses; none of the data points were removed.

\section{Statistical analysis of proteomics data}

For both statistical and network analysis, we used inhouse developed R-software based on STRING [54-57], circlize (https://jokergoo.github.io/circlize_book/book/), MASS [55], lsmeans [56], matrixStats [57], reshape2 [58] and ggplot2 [59] packages. Assuming that data from technical repetitions are often characterized by Poisson distribution [60], and the large variances of biological replicas can be modelled by negative binomial distribution [61], we used modified general linear models to describe group-level differences in measured protein data in the 4 and $12 \mathrm{~h}$ time points. For each protein; after fitting negative binomial generalized linear model [55], we performed a post-hoc analysis [62] to characterize timedependent mean differences by $\mathrm{z}$ score, and corrected $p$ values for multiple comparisons.

\section{Network analysis}

Gene names of the identified human proteins were subjected to STRING database [22] and five PPI networks were generated. The NW0 combined proteins from mock- and HIV-1 plasmid-treated cell lysates collected at 0 time-point, the $\mathrm{C} 04$ and $\mathrm{C} 12$ networks contained proteins from the mock cells collected 4 and $12 \mathrm{~h}$ postinfection, respectively, while the $\mathrm{H} 04$ and $\mathrm{H} 12$ networks contained proteins from the HIV-1 treated cells collected at 4 and $12 \mathrm{~h}$ time-points, respectively. Very high confidence interactions (interaction score $>0.95$ ) in between the query proteins were used for the generation of each binary network. In these networks, the nodes were the proteins and the edges indicated the interactions between proteins as they were present in STRING. For network generation, the SRING R-package and the STRING database was applied, and the 0.95 combined score value to generate the binary networks $B_{t, s}\left(B_{0}\right.$, $\left.\mathrm{B}_{4 \mathrm{~h}, \mathrm{C}}, \mathrm{B}_{4 \mathrm{~h}, \mathrm{H}}, \mathrm{B}_{12 \mathrm{~h}, \mathrm{C}}, \mathrm{B}_{12 \mathrm{~h}, \mathrm{H}}\right)$ corresponding to the protein sets. In these networks, the binary edges indicated only the possibility of the interactions, taking no notice of the quantity.

To estimate the real interaction density, binary networks $\left(B_{t, s}\right)$ generated by STRING were further modified, and the amount of proteins measured by spectral counting was used to add $\mathrm{w}_{\mathrm{ij}}$ weights to the edges. In this way, the existence of edges provides information on the existence of interaction, and the strength of protein pair's interactions were estimated by this edge-weight model:

$$
w_{i j}=\frac{n_{i}}{k_{i}} \frac{n_{j}}{k_{j}}
$$

where $\mathrm{w}_{\mathrm{ij}}$ represents the interaction density between protein $P_{i}$ and $P_{j} ; n_{i}, n_{j}$ means the quantity while $k_{i}, k_{j}$ denote the degree (the number of edges) of $P_{i}$ and $P_{j}$ in the given $\mathrm{B}_{\mathrm{t}, \mathrm{s}}$ binary network.

In this calculation, we used the measured data $\left(n_{i}, n_{j}\right)$, which enabled us to alter the theoretical binary PPI 
network into a realistic, sample related interaction network, in which the weights of the edges are in direct proportion to the quantities and in inverse proportion to all interaction possibilities of the connected proteins in the given sample.

Because we can consider the $\mathrm{n}_{\mathrm{i}}$ as the number of molecules of the protein $\mathrm{P}_{\mathrm{i}}$, the $\mathrm{n}_{\mathrm{i}} / \mathrm{k}_{\mathrm{i}}$ ratio represents the number of $\mathrm{P}_{\mathrm{i}}$ molecules involved in one interaction of $\mathrm{P}_{\mathrm{i}}$, and thus, the interaction density between $P_{i}$ and $P_{j}$ can be described by the product of $n_{i} / k_{i}$ and $n_{j} / k_{j}$. It should be mentioned that the used edge-weight model in the absence of a strong interactor protein may overestimate the effect of other weak interactor proteins, also, interaction strength data cannot be achieved in a classical quantitative proteomics experiment, and currently are unavailable in publicly accessible databases.

\section{Functional subnetwork construction}

In order to investigate the PPI networks of the proteins belonging to GO (geneontology.org) terms, we marked in each $\mathrm{W}_{\mathrm{t}, \mathrm{s}}$ the nodes by a function flag, which indicated whether or not the protein belongs to a given f-function; in our case, to a GO term. The so-called functional enrichment according to GO terms was done by STRING, using default settings and the Molecular Function, Biological Process and Cellular Component GO terms listed as enriched by STRING in C04, H04, C12 and H12, where at least 10 protein per GO function in any of the networks was present, were considered. This procedure defined a sort of $\mathrm{W}^{\mathrm{f}}{ }_{\mathrm{t}, \mathrm{s}}$ functional networks, and divided them into two disjunctive sub-networks $\left(\mathrm{F}^{\mathrm{f}}{ }_{\mathrm{t}, \mathrm{s}}\right.$ functional, belonging to the GO term and $\mathrm{NF}^{\mathrm{f}}{ }_{\mathrm{t}, \mathrm{s}}$ non-functional not being part of the respective GO term), containing the functional and the non-functional nodes, respectively. Because of this separation, the edges (i.e. the interactions) were also classified into three classes: functional edges between the functional nodes, non-functional edges between non-functional nodes and cross-edges in between functional and non-functional nodes, depending on the f-markers of the connected proteins.

\section{Examination of the global characteristics of the evaluated PPI networks}

Any undirected weighted PPI network W(N,E) consists of two sets: $\mathrm{N}$ nodes and $\mathrm{E}$ edges. Each of the links (interactions) is defined by a couple of nodes (proteins) $P_{i}$ and $P_{j}$, and its value is $w_{i j}$. Since the direction of interaction cannot be ordered, the connectivity matrix became symmetric: $\mathrm{w}_{\mathrm{ij}}=\mathrm{w}_{\mathrm{j} \text { i }}$.

\section{Number of nodes $(N)$ and edges $(E)$}

$\mathrm{N}, \mathrm{Nf}$ and $\mathrm{Nn}$ denotes the number of nodes (i.e. proteins) in the whole network and the functional and nonfunctional sub-networks, respectively, with the following relation:

$$
\mathrm{N}=\mathrm{Nf}+\mathrm{Nn}
$$

$E$ denotes the number of edges (i.e. interactions) in the whole network. Ef and En are the number of edges within the functional and the non-functional subnetworks, respectively. The number of cross-edges $(\mathrm{Ec})$ shows the connected proteins between the functional and the non-functional sub-networks. The edge numbers follow the next relation:

$$
E=E f+E n+E c
$$

\section{Network strength and averaged node strength (S)}

We defined the network strength $S$ as the total sum of the weights of edges:

$$
S=\frac{1}{2} \sum_{i, j=1}^{N} w_{i, j}
$$

In the functional networks we can calculate strength of whole network (S), and the functional (Sf) and nonfunctional sub-networks (Sn), as well. The sum of cross connection edges can be calculate as follows:

$$
\mathrm{Sc}=\mathrm{S}-\mathrm{Sf}-\mathrm{Sn}
$$

\section{Edge-weight density or strength density (D)}

the edge-weight density measures how the weighted network is saturated by strong edges:

$$
D=\frac{S}{w_{\max } \frac{N(N-1)}{2}}
$$

In the functional networks we can measure the edgeweight density of the whole network (D) and the functional (Df) and non-functional sub-networks (Dn), as well.

Edge-weight ratio ( $\mathrm{R})$ : using the network strength we can define the edge-weight ratio parameter for the two sub-networks:

$$
R f=\frac{S f}{S}
$$

and the non-functional relative edge-weight density:

$$
R n=\frac{S n}{S}
$$

Since the distribution of network parameters was not Gaussian or negative binomial, we used Wilcoxson tests [63] to characterize the group-related differences at the 4 and $12 \mathrm{~h}$ time points. The evaluated $p$-values were corrected for multiple comparisons by false discovery rate methods [64]. 


\section{Additional files}

Additional file 1: List of identified proteins. (XLSX $1147 \mathrm{~kb}$ )

Additional file 2: List of quantified proteins. The gene name according to UniProt in case of quantified proteins is given, and for each protein, the mean amount of four replicates is presented for each time point except NW, where the mean amount of 8 replicates is given. (XLSX 23 $\mathrm{kb})$

Additional file 3: Statistical analysis of protein quantities. The gene name according to UniProt, the $p$ value and $\mathrm{z}$ score for the $4 \mathrm{~h}$ and $12 \mathrm{~h}$ time points are listed in case of each protein. The lists are presented in ascending order of the $p$ values. (XLSX $24 \mathrm{~kb})$

Additional file 4: Network parameters calculated for functional subnetworks. The $y$ axis show the mean value characteristic for each parameter, and the $x$ axis indicates the time points. Blue color refers to the control, while the yellow color to the HIV-1 treated conditions. $\mathrm{N}$ refers to the number of nodes, $\mathrm{E}$ to the number of edges, $\mathrm{S}$ show network strength, $D$ represents the edge density and $R$ the edge ratio. The f refers to the functional sub-network, the $\mathrm{n}$ to the non-functional subnetwork containing the proteins not present in the functional sub-network, while the $c$ refers to the interactions between the functional and the nonfunctional sub-networks. (PDF $9676 \mathrm{~kb}$ )

Additional file 5: Statistical analysis of network parameters. The FDRcorrected $\mathrm{p}$ value and $\mathrm{z}$ score for the $4 \mathrm{~h}$ and $12 \mathrm{~h}$ time points, respectively, in case of each network parameter calculated (N, Nf, Nn, E, Ef, En, Ec, S, Sf, Sn, Sc, D, Df, Dn, Dc, Rf, Rn, Rc) for each GO function presented in Additional file 4. (XLSX $287 \mathrm{~kb}$ )

Additional file 6: List of $\mathrm{GO}$ terms with network parameters that were significantly changed in the functional sub-networks. (XLSX $16 \mathrm{~kb}$ )

\section{Abbreviations}

cDNA: Complementary DNA; D: Saturation of the PPI interaction density in the sample; Dc: Saturation of the PPI interaction density between the functional and non-functional proteins in the sample; Df: Saturation of the PPI interaction density of the functional proteins in the sample; DMEM: Dulbecco's modified Eagle's medium; Dn: Saturation of the PPI interaction density of the nonfunctional proteins in the sample; DNA: Deoxyribonucleic acid; E: Number of interactions in the network generated from STRING with combined score of 0.95; Ec: Number of interactions between the functional and non-functional proteins; Ef: Number of interactions between the functional proteins; En: Number of interactions between the non-functional proteins; FBS: Fetal bovine serum; GO: Gene Ontology; HEK: Human embryonic kidney cells; HIV: Human immunodeficiency virus; MIPS: Monoisotopic precursor selection; N: Number of proteins in the network; Nf: Number of proteins in the functional subnetwork; Nn: Number of proteins in the non-functional sub-network; PBS: Phosphate buffered saline; PPI: Protein-protein interaction; ppm: Parts per million; R: Relative PPI density, edge-weight ratio; Rc: Relative crossfunctional PPI density, edge-weight ratio between the functional and nonfunctional PPI networks; Rf: Relative functional PPI density, edge-weight ratio between in the functional PPI network; Rn: Relative non-functional PPI density, edge-weight ratio in the non-functional PPI network; RNA: Ribonucleic acid; RT: Reverse transcriptase; S: PPI density of the sample; Sc: PPI density of the cross-functional proteins in the sample; Sf: PPI density of the functional proteins in the sample; siRNA: Small interfering RNA; Sn: PPI density of the non-functional proteins in the sample; VSV: Vesicular stomatitis virus

\section{Acknowledgements}

Not applicable.

\section{Authors' contributions}

JT conceptualized the study, designed the HIV-1 transduction experiments, reviewed the manuscript and provided the resources for the study. FT produced the viruses, carried out the cell transductions, prepared the samples for mass spectrometry and curated the mass spectrometry data. EC designed the data analysis strategy, analyzed the mass spectrometry, statistical and network analysis data, prepared the tables and wrote the manuscript. GT performed the mass spectrometry experiment and helped with the manuscript preparation. ME designed and performed the statistical analysis and network analysis, assisted in manuscript writing and prepared the Figs. MM participated in manuscript preparation, critically reviewed the manuscript, performed the English editing and helped with the evaluation of HIV-1 transduction results. All authors read and approved the final manuscript.

\section{Funding}

This work was supported by the Hungarian Scientific Research Fund (NKFI-6, 125238) to JT, by the Higher Education Institutional Excellence Programme of the Ministry of Human Capacities in Hungary, within the framework of the Biotechnology thematic programme of the University of Debrecen, GINOP2.3.3-15-2016-00020, and partially by Janos Bolyai Research Scholarship of the Hungarian Academy of Sciences. Mass spectrometry data were acquired by the Arizona Proteomics Consortium supported by NIEHS grant ES06694 to the SWEHSC, NIH/NCl grant CA023074 to the UA Cancer Center and by the $\mathrm{BIO5}$ Institute of the University of Arizona. The Thermo Fisher LTQ Orbitrap Velos mass spectrometer was provided by grant 1S10 RR028868-01 from $\mathrm{NIH} / \mathrm{NCRR}$. Funding bodies did not play any roles in the design of the study and collection, analysis, and interpretation of data and in writing the manuscript.

\section{Availability of data and materials}

The mass spectrometry datasets generated during the current study were deposited to the ProteomeXchange database and are available via the PRIDE repository with the dataset identifier PXD010436 and https://doi.org/10.6019/ PXD010436. All data analyzed during this study are included in this published article [and its supplementary information files].

\section{Ethics approval and consent to participate} Non applicable.

\section{Consent for publication}

Not applicable.

\section{Competing interests}

The authors declare that they have no competing interests.

\section{Author details}

${ }^{1}$ Proteomics Core Facility, Department of Biochemistry and Molecular Biology, Faculty of Medicine, University of Debrecen, Egyetem ter 1., Debrecen 4032, Hungary. ${ }^{2}$ Laboratory of Retroviral Biochemistry, Department of Biochemistry and Molecular Biology, Faculty of Medicine, University of Debrecen, Egyetem ter 1., Debrecen 4032, Hungary. ${ }^{3}$ Arizona Research Labs, University of Arizona, PO Box 210066, Administration Building, Room 601, Tucson, AZ 85721-0066, USA. ${ }^{4}$ The Scripps Research Institute, 132 Scripps Way, Jupiter, FL 33458, USA. ${ }^{5}$ Department of Medical Imaging, Division of Nuclear Medicine and Translational Imaging, Faculty of Medicine, University of Debrecen, Nagyerdei krt. 98., Debrecen 4032, Hungary.

Received: 7 May 2019 Accepted: 10 July 2019

Published online: 17 July 2019

\section{References}

1. Keller A, Nesvizhskii Al, Kolker E, Aebersold R. Empirical statistical model to estimate the accuracy of peptide identifications made by MS/MS and database search. Anal Chem. 2002;74(20):5383-92.

2. Domon B, Aebersold R. Options and considerations when selecting a quantitative proteomics strategy. Nat Biotechnol. 2010;28(7):710-21.

3. Codrea MC, Nahnsen S. Platforms and pipelines for proteomics data analysis and management. Modern Proteomics - Sample Preparation, Analysis and Practical Applications. 2016;919:203-15.

4. Kentaro Kawata AH, Yugi K, Kubota H, Sano T, Fujii M, Tomizawa Y, Kokaji T, Tanaka KY, Uda S, Yutaka S, Matsumoto M, Nakayama Kl, Saitoh K, Kato K, Ueno A, Ohishi M, Hirayama A, Kuroda S. Trans-omic Analysis Reveals Selective Responses to Induced and Basal Insulin across Signaling, Transcriptional, and Metabolic Networks. iscience. 2018;7:1-18.

5. Koberlin MS, Snijder B, Heinz LX, Baumann CL, Fauster A, Vladimer Gl, Gavin AC, Superti-Furga G. A conserved circular network of Coregulated lipids modulates innate immune responses. Cell. 2015;162(1):170-83. 
6. Oldham MC, Konopka G, Iwamoto K, Langfelder P, Kato T, Horvath S, Geschwind DH. Functional organization of the transcriptome in human brain. Nat Neurosci. 2008;11(11):1271-82.

7. Li D, Li YP, Li YX, Zhu XH, Du XG, Zhou M, Li WB, Deng HY. Effect of regulatory network of exosomes and microRNAs on neurodegenerative diseases. Chin Med J. 2018;131(18):2216-25.

8. Szilagyi A, Nussinov R, Csermely P. Allo-network drugs: extension of the allosteric drug concept to protein- protein interaction and signaling networks. Curr Top Med Chem. 2013;13(1):64-77.

9. Jager S, Cimermancic P, Gulbahce N, Johnson JR, McGovern KE, Clarke SC, Shales M, Mercenne G, Pache L, Li K, et al. Global landscape of HIV-human protein complexes. Nature. 2011;481(7381):365-70.

10. Csermely P, Sandhu KS, Hazai E, Hoksza Z, Kiss HJ, Miozzo F, Veres DV, Piazza $F$, Nussinov R. Disordered proteins and network disorder in network descriptions of protein structure, dynamics and function: hypotheses and a comprehensive review. Curr Protein Pept Sci. 2012;13(1):19-33.

11. Dai LY, Zhao TY, Bisteau X, Sun WD, Prabhu N, Lim YT, Sobota RM, Kaldis P, Nordlund P. Modulation of Protein-Interaction States through the Cell Cycle. Cell. 2018;173(6):1481.

12. Weiss RA. The discovery of endogenous retroviruses. Retrovirology. 2006;3:67.

13. Kirchhoff F: HIV Life Cycle: Overview. In: Encyclopedia of AIDS. Edited by Hope TJ, Stevenson M, Richman D. New York, NY: Springer New York; 2013: 1-9.

14. Lehmann-Che J, Saib A. Early stages of HIV replication: how to hijack cellular functions for a successful infection. AIDS Rev. 2004;6(4):199-207.

15. Brass AL, Dykxhoorn DM, Benita Y, Yan N, Engelman A, Xavier RJ, Lieberman J, Elledge SJ. Identification of host proteins required for HIV infection through a functional genomic screen. Science. 2008;319(5865):921-6.

16. Konig R, Zhou Y, Elleder D, Diamond TL, Bonamy GM, Irelan JT, Chiang CY, Tu $B P$, De Jesus PD, Lilley CE, et al. Global analysis of host-pathogen interactions that regulate early-stage HIV-1 replication. Cell. 2008;135(1):49-60.

17. Fu W, Sanders-Beer BE, Katz KS, Maglott DR, Pruitt KD, Ptak RG. Human immunodeficiency virus type 1, human protein interaction database at NCBI. Nucleic Acids Res. 2009;37:D417-22.

18. MacPherson JI, Dickerson JE, Pinney JW, Robertson DL. Patterns of HIV-1 protein interaction identify perturbed host-cellular subsystems. PLoS Comput Biol. 2010;6(7):e1000863.

19. Dickerson JE, Pinney JW, Robertson DL. The biological context of HIV-1 host interactions reveals subtle insights into a system hijack. BMC Syst Biol. 2010; 4:80.

20. Pinney JW, Dickerson JE, Fu W, Sanders-Beer BE, Ptak RG, Robertson DL. HIV-host interactions: a map of viral perturbation of the host system. Aids. 2009;23(5):549-54.

21. Vizcaino JA, Deutsch EW, Wang R, Csordas A, Reisinger F, Rios D, Dianes JA, Sun Z, Farrah T, Bandeira N, et al. ProteomeXchange provides globally coordinated proteomics data submission and dissemination. Nat Biotechnol. 2014;32(3):223-6.

22. Szklarczyk D, Morris JH, Cook H, Kuhn M, Wyder S, Simonovic M, Santos A, Doncheva NT, Roth A, Bork P, et al. The STRING database in 2017: qualitycontrolled protein-protein association networks, made broadly accessible. Nucleic Acids Res. 2017;45(D1):D362-8.

23. Zhou HL, Xu M, Huang Q, Gates AT, Zhang XHD, Castle JC, Stec E, Ferrer M, Strulovici B, Hazuda DJ, et al. Genome-scale RNAi screen for host factors required for HIV replication. Cell Host Microbe. 2008;4(5):495-504.

24. Arhel N, Kirchhoff F. Host proteins involved in HIV infection: new therapeutic targets. Bba-Mol Basis Dis. 2010;1802(3):313-21.

25. Mohammadi P, Desfarges S, Bartha I, Joos B, Zangger N, Munoz M, Gunthard HF, Beerenwinkel N, Telenti A, Ciuffi A. 24 hours in the life of HIV1 in a T cell line. PLoS Pathog. 2013;9(1):e1003161.

26. Nemeth J, Vongrad V, Metzner KJ, Strouvelle VP, Weber R, Pedrioli P, Aebersold R, Gunthard HF, Collins B. In vivo and in vitro proteome analysis of human immunodeficiency virus (HIV)-1-infected, human CD4(+) T cells. Mol Cell Proteomics. 2017;16(4):S108-23.

27. Goff SP. Knockdown screens to knockout HIV-1. Cell. 2008;135(3):417-20.

28. Yeung ML, Houzet L, Yedavalli VSRK, Jeang KT. A genome-wide short hairpin RNA screening of Jurkat T-cells for human proteins contributing to productive HIV-1 replication. J Biol Chem. 2009;284(29):19463-73.

29. de Lichtenberg U, Jensen $L$, Brunak S, Bork P. Dynamic complex formation during the yeast cell cycle. Science. 2005;307(5710):724-7.

30. Greene CS, Krishnan A, Wong AK, Ricciotti E, Zelaya RA, Himmelstein DS, Zhang R, Hartmann BM, Zaslavsky E, Sealfon SC, et al. Understanding multicellular function and disease with human tissue-specific networks. Nat Genet. 2015;47(6):569-76.
31. Celaj A, Schlecht U, Smith JD, Xu W, Suresh S, Miranda M, Aparicio AM, Proctor M, Davis RW, Roth FP, et al. Quantitative analysis of protein interaction network dynamics in yeast. Mol Syst Biol. 2017;13(7):934.

32. Sardiu ME, Cai Y, Jin J, Swanson SK, Conaway RC, Conaway JW, Florens L, Washburn MP. Probabilistic assembly of human protein interaction networks from label-free quantitative proteomics. Proc Natl Acad Sci U S A. 2008;105(5):1454-9.

33. Boccaletti S, Latora V, Moreno Y, Chavez M, Hwang DU. Complex networks: structure and dynamics. Phys Rep. 2006;424(4-5):175-308.

34. Kleinman CL, Doria M, Orecchini E, Giuliani E, Galardi S, De Jay N, Michienzi A. HIV-1 infection causes a down-regulation of genes involved in ribosome biogenesis. PloS one. 2014;9(12):e113908.

35. Dowling D, Nasr-Esfahani S, Tan CH, O'Brien K, Howard JL, Jans DA, Purcell DF, Stoltzfus CM, Sonza S. HIV-1 infection induces changes in expression of cellular splicing factors that regulate alternative viral splicing and virus production in macrophages. Retrovirology. 2008;5:18.

36. Sertznig $H$, Hillebrand F, Erkelenz S, Schaal H, Widera M. Behind the scenes of HIV-1 replication: Alternative splicing as the dependency factor on the quiet. Virology. 2018;516:176-188

37. Garcia-Moreno M, Noerenberg M, Ni S, Jarvelin Al, Gonzalez-Almela E, Lenz CE, Bach-Pages M, Cox V, Avolio R, Davis T, et al. System-wide Profiling of RNA-Binding Proteins Uncovers Key Regulators of Virus Infection. Molecular cell. 2019;74(1):196-211, e111.

38. Lum JJ, Badley AD: Resistance to apoptosis: mechanism for the development of HIV reservoirs. Current HIV research. 2003;1(3):261-274.

39. Antoni $B A$, Sabbatini $P$, Rabson $A B$, White E. Inhibition of apoptosis in human immunodeficiency virus-infected cells enhances virus production and facilitates persistent infection. J Virol. 1995;69(4):2384-392.

40. Badley AD, Sainski A, Wightman F, Lewin SR. Altering cell death pathways as an approach to cure HIV infection. Cell death \& disease. 2013;4:e718.

41. Stricher F, Macri C, Ruff M, Muller S. HSPA8/HSC70 chaperone protein: structure, function, and chemical targeting. Autophagy. 2013;9(12):1937-54.

42. Sherman MP, Greene WC. Slipping through the door: HIV entry into the nucleus. Microbes and infection / Institut Pasteur. 2002;4(1):67-73.

43. Chang ST, Sova P, Peng X, Weiss J, Law GL, Palermo RE, Katze MG. Nextgeneration sequencing reveals HIV-1-mediated suppression of T cell activation and RNA processing and regulation of noncoding RNA expression in a CD4+ T cell line. mBio. 2011;2(5).

44. Rato $\mathrm{S}$, Rausell A, Munoz M, Telenti A, Ciuffi A. Single-cell analysis identifies cellular markers of the HIV permissive cell. Plos Pathog. 2017;13(10):e1006678.

45. Rao S, Amorim R, Niu M, Breton Y, Tremblay MJ, Mouland AJ. Host mRNA decay proteins influence HIV-1 replication and viral gene expression in primary monocyte-derived macrophages. Retrovirology. 2019;16(1):3.

46. Tabb DL, Vega-Montoto L, Rudnick PA, Variyath AM, Ham AJ, Bunk DM, Kil patrick LE, Billheimer DD, Blackman RK, Cardasis $\mathrm{HL}$, et al. Repeatability and reproducibility in proteomic identifications by liquid chromatography-tandem mass spectrometry. Journal of proteome research. 2010;9(2):761-76.

47. Heaven MR, Funk AJ, Cobbs AL, Haffey WD, Norris JL, McCullumsmith RE, Greis KD. Systematic evaluation of data-independent acquisition for sensitive and reproducible proteomics-a prototype design for a single injection assay. J Mass Spectrom : JMS. 2016;51(1):1-11.

48. Miklossy G, Tozser J, Kadas J, Ishima R, Louis JM, Bagossi P. Novel macromolecular inhibitors of human immunodeficiency virus-1 protease. Protein engineering, design \& selection : PEDS. 2008;21 (7):453-61.

49. Dull T, Zufferey R, Kelly M, Mandel RJ, Nguyen M, Trono D, Naldini L. A third-generation lentivirus vector with a conditional packaging system. J Virol. 1998;72(11):8463-71.

50. Csosz E, Markus B, Darula Z, Medzihradszky KF, Nemes J, Szabo E, Tozser J, Kiss C, Marton I. Salivary proteome profiling of oral squamous cell carcinoma in a Hungarian population. FEBS open bio. 2018;8(4):556-69.

51. Andon NL, Hollingworth S, Koller A, Greenland AJ, Yates JR 3rd, Haynes PA. Proteomic characterization of wheat amyloplasts using identification of proteins by tandem mass spectrometry. Proteomics. 2002;2(9):1156-68.

52. Qian WJ, Liu T, Monroe ME, Strittmatter EF, Jacobs JM, Kangas LJ, Petritis K, Camp DG 2nd, Smith RD. Probability-based evaluation of peptide and protein identifications from tandem mass spectrometry and SEQUEST analysis: the human proteome. J Proteome Res. 2005;4(1):53-62.

53. Nesvizhskii Al, Keller A, Kolker E, Aebersold R. A statistical model for identifying proteins by tandem mass spectrometry. Analytical chemistry. 2003; 75(17):4646-58,

54. Franceschini A, Szklarczyk D, Frankild S, Kuhn M, Simonovic M, Roth A, Lin J, Minguez $P$, Bork P, von Mering C, et al. STRING v9.1: protein-protein 
interaction networks, with increased coverage and integration. Nucleic Acids Res. 2013;41(Database issue):D808-15.

55. W.N. Venables BDR. Modern applied statistics with S. New York: Springer-Verlag; 2002.

56. Lenth RV. Least-squares means: the R package Ismeans. J Stat Softw. 2016;69(1):1-33.

57. matrixStats: Functions that Apply to Rows and Columns of Matrices (and to Vectors). R package version 0.52.2 [https://github.com/ HenrikBengtsson/matrixStats].

58. Wickham H. Reshaping data with the reshape package. J Stat Softw. 2007;21(12):1-20.

59. Ginestet C. ggplot2: elegant graphics for data analysis. J R Stat Soc a Stat. 2011;174:245.

60. Marioni JC, Mason CE, Mane SM, Stephens M, Gilad Y. RNA-seq: an assessment of technical reproducibility and comparison with gene expression arrays. Genome Res. 2008;18(9):1509-17.

61. Robinson MD, Oshlack A. A scaling normalization method for differential expression analysis of RNA-seq data. Genome Biol. 2010;11:3.

62. Searle SR, Speed FM, Milliken GA. Population marginal means in the linearmodel - an alternative to least-squares means. Am Stat. 1980;34(4):216-21.

63. MHaDA W. Nonparametric statistical methods. New York: John Wiley \& Sons; 1999.

64. Benjamini Y, Hochberg Y. Controlling the false discovery rate - a practical and powerful approach to multiple testing. J Roy Stat Soc B Met. 1995;57(1): 289-300.

\section{Publisher's Note}

Springer Nature remains neutral with regard to jurisdictional claims in published maps and institutional affiliations.

- fast, convenient online submission

- thorough peer review by experienced researchers in your field

- rapid publication on acceptance

- support for research data, including large and complex data types

- gold Open Access which fosters wider collaboration and increased citations

- maximum visibility for your research: over $100 \mathrm{M}$ website views per year

At $\mathrm{BMC}$, research is always in progress. 